\title{
Galectin-7 downregulation in lesional keratinocytes contributes to enhanced IL-17A signaling and skin pathology in psoriasis
}

\author{
Hung-Lin Chen, ${ }^{1}$ Chia-Hui Lo, ${ }^{1}$ Chi-Chun Huang, ${ }^{1}$ Meng-Ping Lu, ${ }^{1}$ Po-Yuan Hu, ${ }^{1}$ Chang-Shan Chen, ${ }^{1}$ Di-Yen Chueh, ${ }^{2}$ Peilin Chen, ${ }^{2}$ \\ Teng-Nan Lin, ${ }^{1}$ Yuan-Hsin Lo, ${ }^{3,4}$ Yu-Ping Hsiao, ${ }^{5}$ Daniel K. Hsu, ${ }^{6}$ and Fu-Tong Liu ${ }^{1,6}$ \\ IInstitute of Biomedical Sciences, Academia Sinica, Taipei, Taiwan. ${ }^{2}$ Research Center for Applied Sciences, Academia Sinica, Taipei, Taiwan. ${ }^{3}$ Department of Dermatology, Fu Jen Catholic University Hospital, \\ and ${ }^{4}$ School of Medicine, Fu Jen Catholic University, New Taipei City, Taiwan. ${ }^{5}$ Chung Shan Medical University Hospital, Taichung, Taiwan. ${ }^{6}$ Department of Dermatology, School of Medicine, University of \\ California, Davis, Sacramento, California, USA.
}

\begin{abstract}
Psoriasis is a chronic inflammatory skin disease characterized by inflammatory cell infiltration, as well as hyperproliferation of keratinocytes in skin lesions, and is considered a metabolic syndrome. We found that the expression of galectin-7 is reduced in skin lesions of patients with psoriasis. IL-17A and TNF- $\alpha, 2$ cytokines intimately involved in the development of psoriatic lesions, suppressed galectin-7 expression in human primary keratinocytes (HEKn cells) and the immortalized human keratinocyte cell line HaCaT. A galectin-7 knockdown in these cells elevated the production of IL-6 and IL-8 and enhanced ERK signaling when the cells were stimulated with IL-17A. Galectin-7 attenuated IL-17A-induced production of inflammatory mediators by keratinocytes via the microRNA-146a/ERK pathway. Moreover, galectin-7-deficient mice showed enhanced epidermal hyperplasia and skin inflammation in response to intradermal IL-23 injection. We identified fluvastatin as an inducer of galectin-7 expression by connectivity map analysis, confirmed this effect in keratinocytes, and demonstrated that fluvastatin attenuated IL-6 and IL-8 production induced by IL-17A. Thus, we validate a role of galectin-7 in the pathogenesis of psoriasis, in both epidermal hyperplasia and keratinocyte-mediated inflammatory responses, and formulate a rationale for the use of statins in the treatment of psoriasis.
\end{abstract}

\section{Introduction}

Psoriasis is a complex inflammatory skin disease of which both genetic and environmental factors participate in the pathogenesis. Important characteristics of this disease include keratinocyte hyperproliferation and leukocyte infiltration. Although the etiology is not clear, hyperactive keratinocytes and immune cells are key players in the pathogenesis of psoriasis, and the crosstalk between these cells contributes to the pathological phenotype. Moreover, psoriasis is considered as a metabolic syndrome, associated with dyslipidemia and an increased risk of cardiovascular disease.

In psoriasis, IL-17 cytokines secreted by activated $\mathrm{T}$ cells are the key mediators that coordinate local tissue inflammation. IL-17A induces keratinocyte hyperproliferation and can cause keratinocytes and other cell types to produce proinflammatory cytokines and neutrophil-mobilizing chemokines (including IL-6, CXCL8/IL-8, and CXCL2). The expression of these cytokines and chemokines is dependent on the I $\kappa$ B kinase- $\alpha$ (IKK- $\alpha) / \mathrm{NF}-\kappa \mathrm{B}$ and ERK signaling pathways. Therefore, factors modulating the sensi-

Authorship note: HLC and CHL contributed equally to this work.

Conflict of interest: FTL, HLC, and PCC are inventors on patent application numbers US 16/653,986 and Taiwan 108137064.

Copyright: @ 2021, American Society for Clinical Investigation.

Submitted: June 3, 2019; Accepted: October 2, 2020; Published: January 4, 2021.

Reference information: / Clin Invest. 2021;131(1):e130740.

https://doi.org/10.1172/JCl130740. tivity of keratinocytes to IL-17 through the above signaling pathways may play a crucial role in the pathogenesis of psoriasis.

Galectin-7, a $15-\mathrm{kDa} \beta$-galactoside-binding protein, is highly expressed in epidermal keratinocytes and is induced by the tumor suppressor p53 (1). Galectin-7 is primarily an intracellular protein and can be located in both the nucleus and the cytoplasm of keratinocytes $(2,3)$, although it also exists outside the cell and is capable of binding to various glycoconjugates in the extracellular space. Downregulation and nuclear translocation of galectin-7 in transformed and UV light-irradiated keratinocytes have been reported, suggesting that galectin-7 may participate in cellular homeostasis $(2,4)$. In mice, galectin-7 was reported to regulate skin homeostasis during injury, but the detailed mechanism is not clear (5). Previously, we have investigated the role of galectin-7 in the regulation of keratinocyte proliferation (6), but how galectin-7 functions in keratinocytes in the inflammatory response occurring in human inflammatory skin diseases, such as psoriasis, is not clear.

A number of microRNAs (miRNAs) were found to be differentially expressed in psoriatic lesions (7). According to a number of mechanistic studies, miRNAs can drive various pathways in psoriasis pathogenesis, including regulation of $\mathrm{T}$ cell activation and functions, IFN- $\alpha$ and TNF- $\alpha$ expression, keratinocyte hyperproliferation, cytokine or chemokine expression, and NF- $\mathrm{BB}$ signaling (8-11). We have previously identified 2 miRNAs that are regulated by galectin-7, miR-146a, and miR-203 (6). In that report, we showed that galectin-7 protects JNK1 from ubiquitination and degradation, which in turn upregulates expression of miR-203. 
We also demonstrated that miR-203 upregulates the expression of p63, which is known to suppress keratinocyte proliferation. Thus, galectin-7 suppresses keratinocyte proliferation through the JNK/ miR-203/p63 pathway.

In the data obtained in our previous transcriptomic study (12), we found that galectin-7 mRNA is downregulated in skin lesions of patients with psoriasis (GEO GSE153007). In the present study, we first explored the protein levels of galectin-7 in psoriasis and then provided evidence that its downregulation in keratinocytes, as noted in psoriatic lesions, contributes to the inflammatory response and epidermal hyperplasia associated with psoriasis. We then dissected the underlying mechanism and established that galectin-7 suppresses the inflammatory response associated with psoriasis by suppressing the miRNA-146a/ERK pathway in keratinocytes. Furthermore, we identified a mechanistic basis for the use of statins, commonly used in the treatment of dyslipidemia, as therapeutic agents in psoriasis attributable to their potent induction of galectin-7 expression.

\section{Results}

Galectin-7 expression is downregulated in human psoriatic lesions and mouse psoriasiform dermatitic lesions induced by $I L-23$. Consistent with the data from our previous transcriptomic study (12) (GEO GSE153007), meta-analysis of the microarray and RNA sequencing data sets (GEO GSE68937 and SRA PRJNA421744) from other publications $(13,14)$ revealed that galectin-7 mRNA is downregulated in the epidermis of skin lesions of patients with psoriasis (Supplemental Tables 1 and 2; supplemental material available online with this article; https://doi.org/10.1172/JCI130740DS1). To confirm this finding at the protein level, we examined galectin-7 protein expression in skin lesions from a cohort of patients with psoriasis $(n=27)$ and normal skin from healthy controls $(n=$ 75) by immunohistochemistry. As shown by representative images (Figure 1A), galectin-7 was strongly expressed in all epidermal layers of normal skin (Figure 1A), but significantly downregulated in psoriatic skin (Figure 1A). The reduction in the galectin-7 protein levels in psoriatic epidermis was verified by computer-assisted quantitative analysis of immunohistochemical staining (Figure 1B). In addition, we confirmed that galectin-7 protein expression was reduced in lesional skin compared with nonlesional skin from the same patients $(n=10)$ (Figure $1 C)$, and the quantification results are shown in Figure 1D. These immunohistochemical analyses (Figure 1, A and C) showed that galectin-7 is expressed mainly in the epidermis and is undetectable in the dermis. Moreover, we performed immunoblot analysis with samples from lesional and nonlesional skin (epidermal plus dermis) of 3 psoriasis patients. There, we confirmed that galectin-7 protein levels were significantly lower in the lesional skin compared with the nonlesional skin (Supplemental Figure 1). It is to be noted that an earlier study (15) did not observe reduced expression of galectin-7 in psoriasis lesions, which could be due to a number of possibilities, including the population of patients (thus the severity of the disease), the antibodies used, and the tissue preservation methods.

We next addressed whether galectin-7 expression was also reduced in a mouse model of psoriasis that involves intradermal IL-23 injections $(16,17)$. Injection of IL-23 into the ears of WT mice induced marked ear swelling, as expected. This response was associated with epidermal hyperplasia and leukocyte infiltration, which were not seen with PBS injection (controls; Figure 1C). IL-23-injected epidermis also showed drastically reduced galectin-7 expression (Figure 1E), as quantified in Figure 1F.

We then examined the effects of cytokines, e.g., IL-17A, IL-23, and TNF- $\alpha$, which are known to promote psoriatic inflammation (17-19), on galectin-7 expression in immortalized human keratinocytes (HaCaT cells) and primary neonatal epidermal keratinocytes (HEKn cells). We found that IL-17A alone reduced galectin-7 protein expression in both (Figure $1 \mathrm{G}$ and Supplemental Figure 2). TNF- $\alpha$ also significantly reduced galectin-7 expression in HaCaT cells, while IFN- $\gamma$, lipopolysaccharide (LPS), and IL-23 had only modest effects (Figure 1G).

Suppression of galectin-7 expression promotes production of proinflammatory cytokines and chemokines. Microarray analysis of galectin-7-knockdown $\mathrm{HaCaT}$ cells revealed upregulation of several chemokine genes (CCL3, CCL4, CXCL2, CXCL3, and others) and psoriasis-related genes (including serine protease inhibitors, SERPINA3 and SERPINB4; S100 calcium-binding proteins, S100A7 and S100A7A; and defensin $\beta 4 \mathrm{~A}, D E F B 4 A$ ) in these cells, as compared with control cells (our unpublished observations). This finding suggested that reduced galectin-7 expression might promote inflammation, thereby contributing to the pathogenesis of psoriasis. We examined the expression of the cell surface receptors IL-17RA, TLR4, and IFN- $\gamma$ R by flow cytometry, and found no significant changes in galectin-7-knockdown cells compared with controls (our unpublished observations). Accordingly, we hypothesized that galectin-7 has a suppressive effect on the cell signaling pathways of inflammatory responses, i.e., galectin-7 downregulation favors proinflammatory cytokine production during inflammation. We therefore subjected both HaCaT and HEKn cells to different immune stimuli, including IL-17A, IL-21, IL-22, IL-23, TNF- $\alpha$, LPS, or IFN- $\gamma$, and measured cytokine secretion by ELISAs. Among these stimuli, only IL-17A (Figure 2), TNF- $\alpha$, and LPS (our unpublished observations) induced production of IL-6 and IL-8, 2 neutrophil-mobilizing chemokines highly implicated in the pathogenesis of psoriasis. Galectin-7-knockdown cells secreted greater amounts of IL-6 and IL-8 in response to stimulation with IL-17A, as compared with control cells (Figure 2). We also observed stronger constitutive secretion of IL- 6 and IL-8 in galectin-7-knockdown cells without stimulation (Figure 2). In addition, an inhibitor of galectin-7 (lactose) had no observed effect on the production of IL-6 and IL-8 (Supplemental Figure 3 ). Since lactose is not cell-permeable, this result suggests that galectin-7 functions in the intracellular region.

MiR-146a expression in keratinocytes is suppressed by galectin-7 and induced by $I L-17 A$. By microarray analysis, deep sequencing, and real-time PCR analyses, we have previously found that miR-146a was upregulated in galectin-7-knockdown HaCaT cells (6). Here, we showed miR-146a was similarly significantly overexpressed in HEKn cells with a transient galectin-7 knockdown (Supplemental Figure 4A). We performed in situ hybridization using locked nucleic acid-modified (LNA-modified) nucleotide probes and confirmed that miR-146a was also expressed in the epidermis of normal human skin, but only rather weakly (Figure 3A). We found that expression of this miRNA was greatly increased in all epidermal layers of psoriatic lesions com- 
A
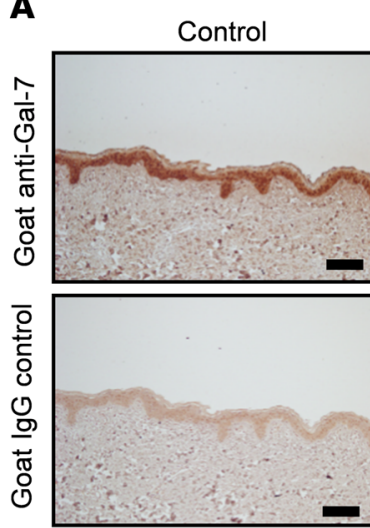

B



$\mathbf{E}$

IL-23-induced psoriasis model

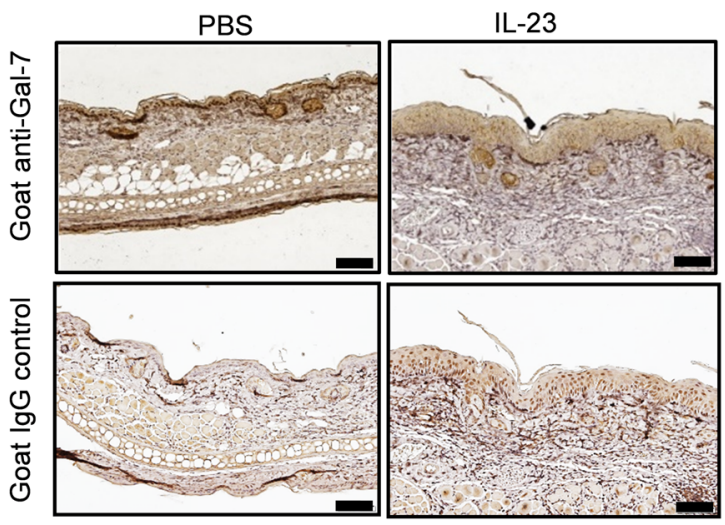

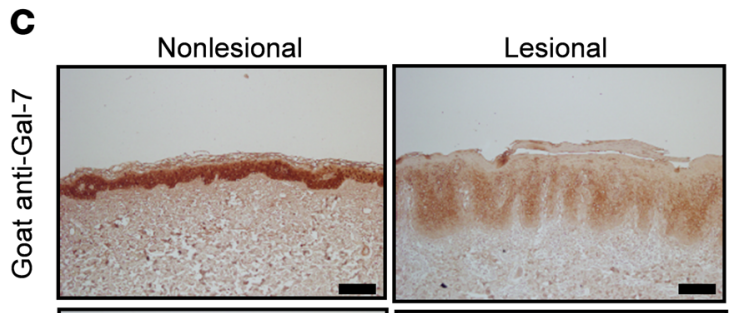
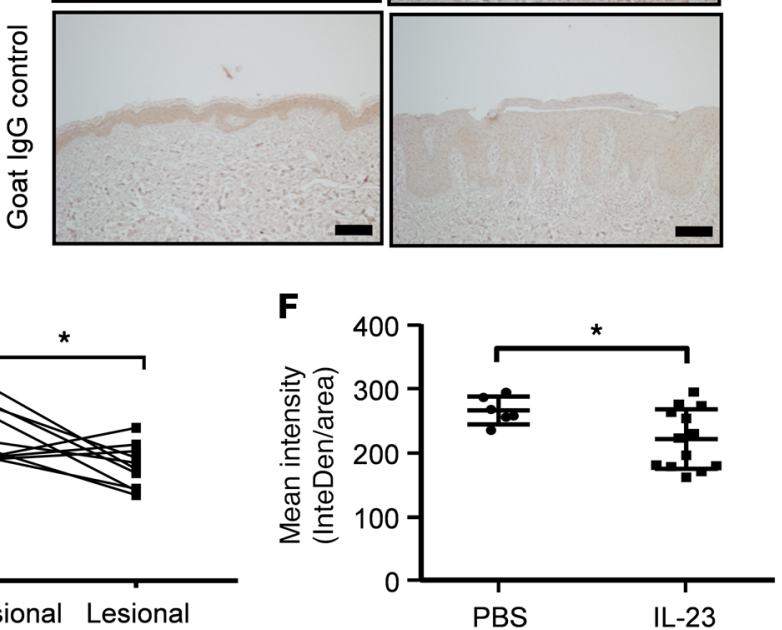

G
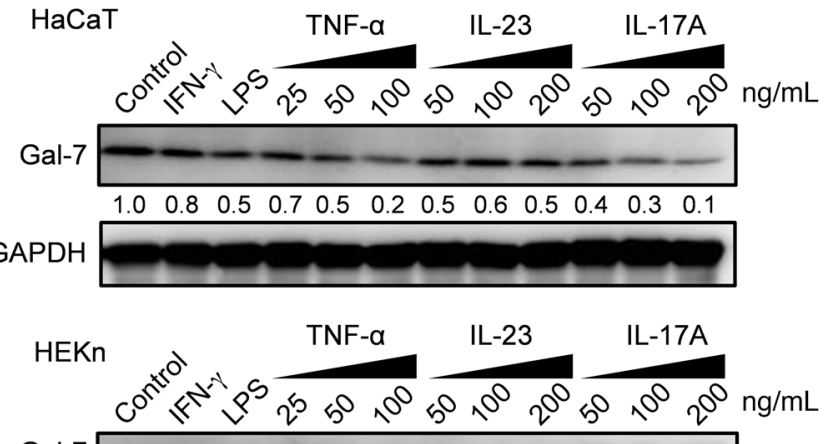

Gal-7

$\begin{array}{llllllllllll}1.0 & 0.9 & 0.8 & 0.7 & 0.7 & 0.5 & 0.2 & 0.5 & 0.3 & 0.3 & 0.2 & 0.1\end{array}$

GAPDH

Figure 1. The levels of galectin-7 are low in the epidermal keratinocytes of patients with psoriasis. (A) Representative immunohistochemical (IHC) staining pictures of galectin-7 in skin sections from a healthy control and a psoriatic lesion. Scale bars: $100 \mu \mathrm{m}$. (B) Quantification of IHC staining of galectin-7 in sections of normal skin from healthy controls $(n=75)$ and sections of lesional skin from patients with psoriasis $(n=27)$. Quantification of staining was performed by computer-assisted methods as described in Methods. The $y$ axis indicates arbitrary numbers representing mean intensity across an area of the epidermis (InteDen/area). (C) IHC staining of galectin-7 in lesional skin and nonlesional skin pair. (D) Quantification of galectin-7 staining of paired lesional and nonlesional skin from the same patients ( $n=10$ pairs). (E) IHC staining of galectin-7 in sections from intradermally IL-23-injected and PBS-injected mouse skin. Scale bars: $100 \mu \mathrm{m}$. (F) Quantification of galectin-7 staining of PBS-injected $(n=6)$ and IL-23-injected mouse skin $(n=13)$. (C) Immunoblot analysis of galectin-7 levels in HaCaT and HEKn cells stimulated with the indicated cytokines (IFN- $\gamma$, LPS, TNF- $\alpha$, IL-23, or IL-17A). The concentrations of IFN- $\gamma$ and LPS used were $100 \mathrm{ng} / \mathrm{mL}$ and $50 \mu \mathrm{g} / \mathrm{mL}$, respectively. The concentrations of TNF- $\alpha$, IL-23, and IL-17A used were as indicated in the figure. Cells were treated with the cytokines for 2 days, and cell lysates were prepared for immunoblot analysis. GAPDH served as a loading control. The intensity of the galectin-7 band was quantified by densitometry and normalized to GAPDH and control samples. The number below each band represents the relative galectin-7 amount. Statistical analyses were performed by unpaired (B and $\mathbf{F}$ ) and paired (D) Student's $t$ test. ${ }^{*} P<0.05$, ${ }^{* * *} P<0.001$.

pared with healthy human skin (Figure 3A). Tissues hybridized with LNA-modified U6 small nuclear RNA-targeting (snRNAtargeting) probes or scrambled probes served as positive and negative controls, respectively (Figure $3 \mathrm{~A}$ ). We treated $\mathrm{HaCaT}$ and HEKn cells with IL-17A and found that miR-146a expression was induced (Figure 3B). In addition, we observed that IL-17A (Fig- ure 3B), but not IL-22 or IL-23 (our unpublished observations), induced miR-146a expression.

To examine the effects of miR-146a under inflammatory conditions, we generated $\mathrm{HaCaT}$ cells stably overexpressing miR-146a (Supplemental Figure 4B). These cells exhibited markedly enhanced production of IL- 6 and IL-8 after IL-17A 
A

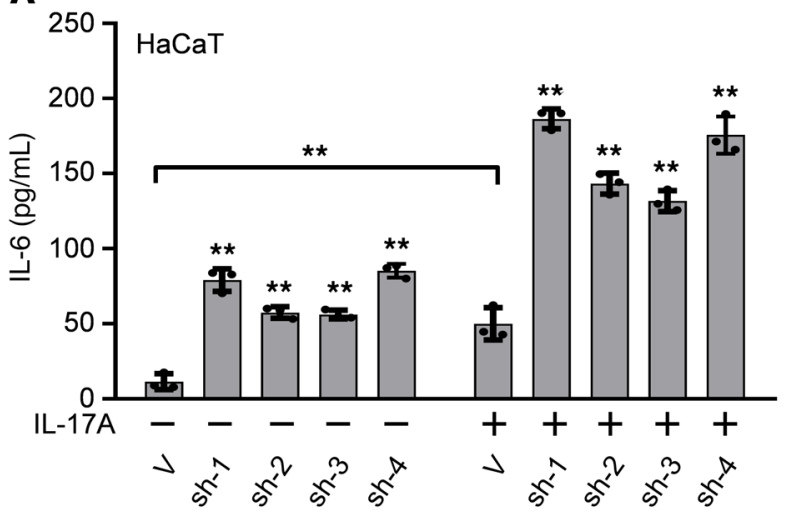

C

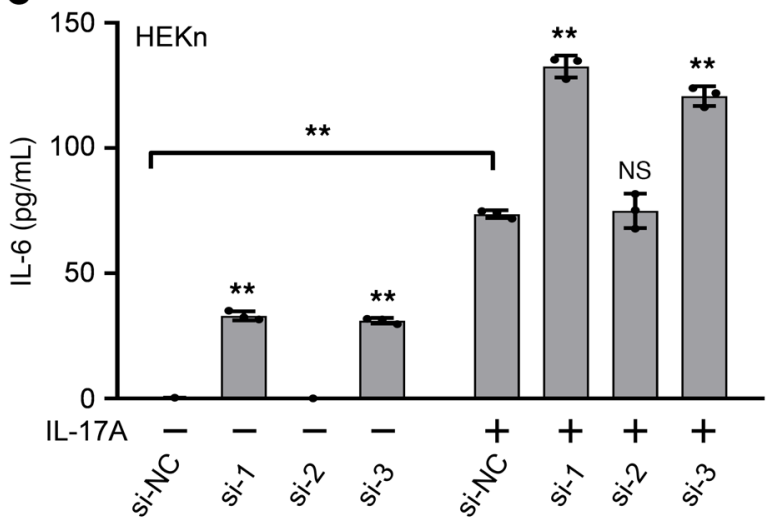

B

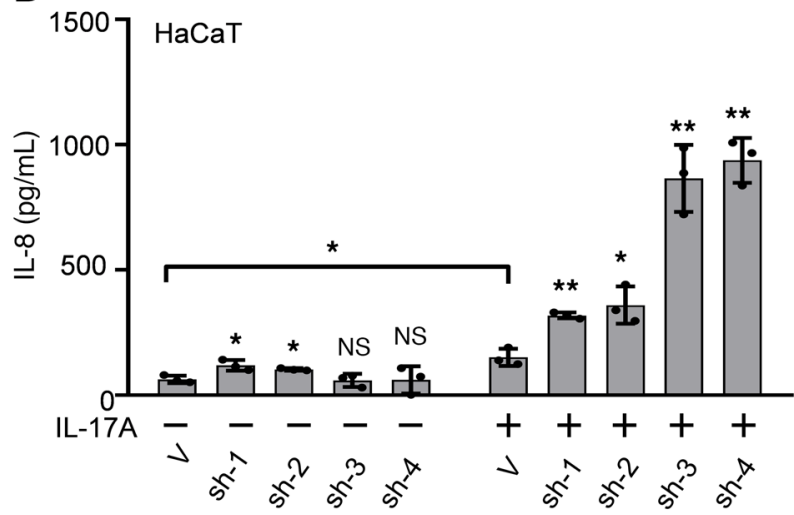

D

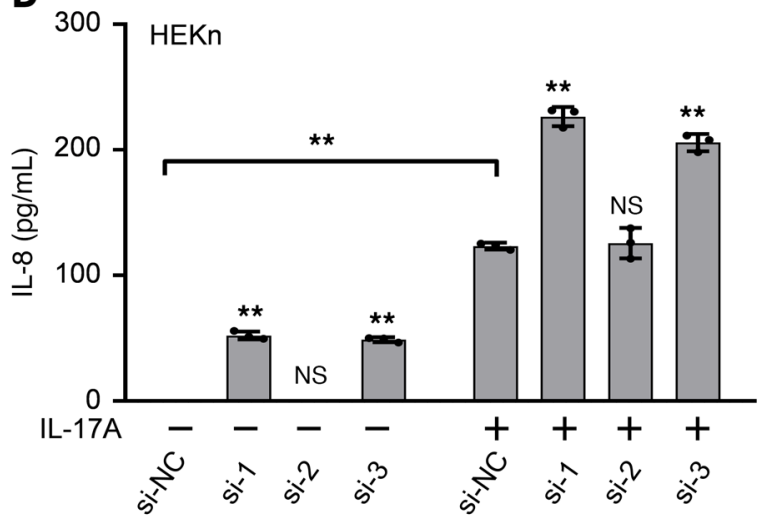

Figure 2. Downregulation of galectin-7 in keratinocytes causes an elevated production of the proinflammatory cytokines IL-6 and IL-8 in response to IL-17A stimulation. (A and B) Galectin-7-knockdown HaCaT cell lines (sh-1, sh-2, sh-3, and sh-4) and controls were incubated with or without IL-17A for 2 days, and IL- 6 and IL-8 concentrations in the supernatants were measured by ELISA. All the experiments included 3 biological replicates. (C and D) HEKn cells were transfected with siRNA to knock down galectin-7 and then incubated with or without IL-17A for 2 days. The IL- 6 and IL-8 concentrations in the supernatants were measured by ELISA. Three independent biological replicates were performed for the ELISA analysis. The results are presented as mean \pm SD. For statistical analysis, 2-way ANOVA with Tukey's multiple-comparison test was performed. Each shRNA- or siRNA-treated cell line was compared with its corresponding control ( $V$, vector; si-NC, negative control siRNA) for both untreated and IL-17-treated groups. The shRNAs and siRNAs for the knockdown of galectin-7 expression were as described in Methods. ${ }^{*} P<0.05,{ }^{* *} P<0.01$.

stimulation, as compared with control cells (Figure 3, C and D), although their galectin-7 expression remained unaffected (Supplemental Figure 4C).

Galectin-7 knockdown enhances the IL-17-induced MAPK/ERK signaling pathway. In response to IL-17A exposure, both the MAPK and NF- $\mathrm{\kappa B}$ pathways participate in the production of proinflammatory cytokines in keratinocytes $(20,21)$. To further dissect the mechanism underlying the involvement of galectin-7 in psoriasis pathogenesis, we focused on the IL-17A signaling pathway. To distinguish whether galectin-7 affects MAPK or NF- $\mathrm{kB}$ activation, we starved $\mathrm{HaCaT}$ cells overnight in serum-free medium and then incubated the cells in the presence or absence of IL-17A. By immunoblotting analysis, we found that resting cells contained small amounts of phosphorylated extracellular signal-regulated kinase 1 (phospho-ERK1) and phospho-ERK2 (Figure 4A). In contrast, exposure of cells to IL-17A induced strong phosphorylation of ERK1 and ERK2 in 4 galectin-7-knockdown cell lines (sh-1, sh-2, sh-3, and sh-4), with 2.0- to 6.6-fold increases compared with control cells (Figure 4A). The total ERK1 and ERK2 protein levels were comparable in all cells treated versus not treated with IL-17A (Figure 4A and Supplemental Figure 5).
In contrast, the upstream components of the NF- $\mathrm{kB}$ pathway

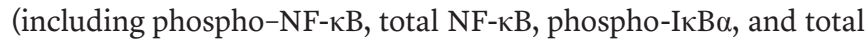
I $\mathrm{KB} \alpha$ ) remained unchanged in response to IL-17A (Figure 4A), suggesting that galectin-7 does not mediate activation of the NF- $\mathrm{\kappa B}$ pathway. We then used specific chemical inhibitors, including inhibitors of the MAP kinases p38 (SB203580), JNK (SP600125), NF-KB (PDTC), PI3K (LY294002), and MAPK/ERK (PD98059), to target their corresponding pathways in galectin-7-knockdown keratinocytes after stimulation with IL-17A. Among these compounds, the MAPK/ERK inhibitor PD98059 significantly blocked IL- 6 and IL-8 production induced by IL-17A (Supplemental Figure 6, A and B). IL-17A-induced IL-6 and IL-8 secretion was not blocked by inhibitors of MAP kinases p38 (SB203580), JNK (SP600125), NF-KB (PDTC), or PI3K (LY294002) (our unpublished observations). Our data suggest that the regulatory role of galectin-7 in IL-17A-induced cytokine expression is primarily mediated by its effect on the MAPK/ERK signaling pathway via suppression of ERK phosphorylation.

MiR-146a induces inflammatory mediators in keratinocytes through the MAPK/ERK pathway. To identify the intracellular pathways via which miR-146a increases the expression of proin- 
A

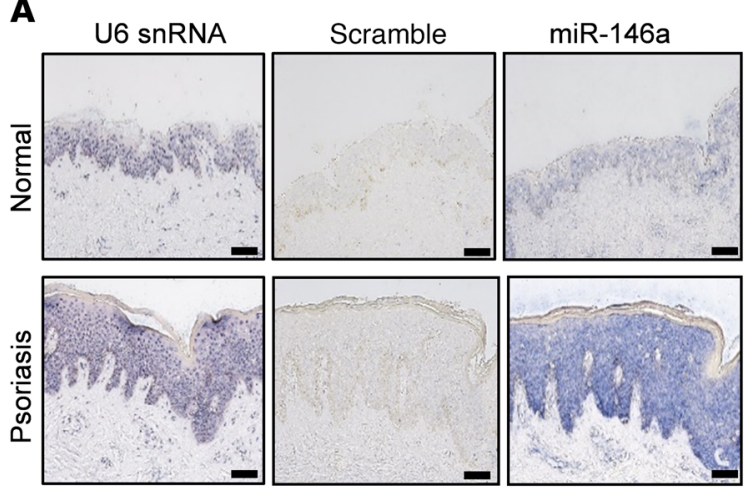

B

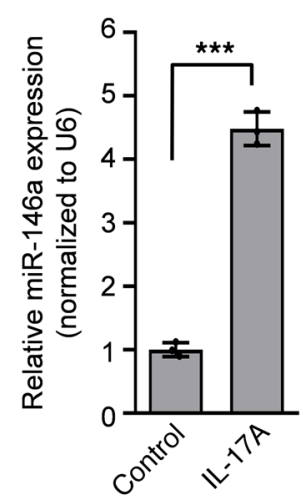

C

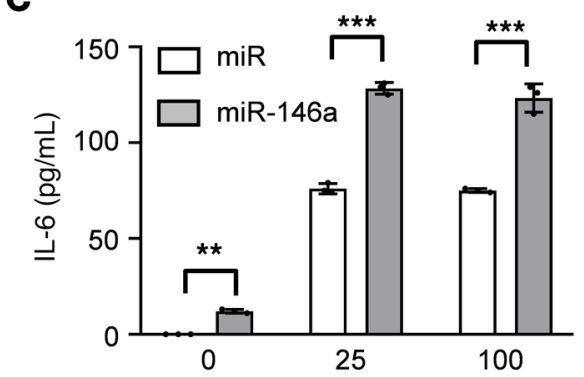

D

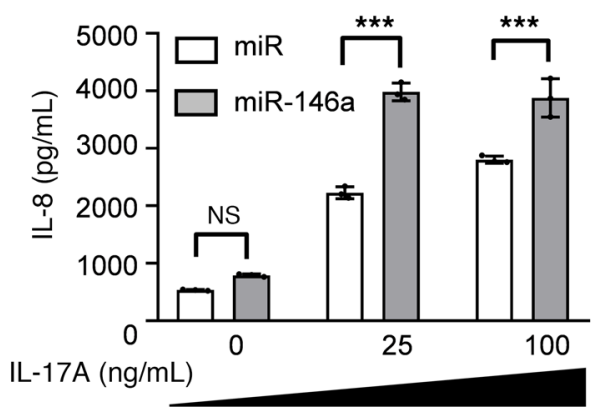

Figure 3. MiR-146a is upregulated in the epidermis of psoriatic lesions; this miRNA promotes production of the proinflammatory cytokines IL-6 and IL-8. (A) MiR-146a in the epidermis of normal and psoriatic skin was detected by RNA in situ hybridization assay. Scale bars: $100 \mu \mathrm{m}$. (B) The miR-146a level in $\mathrm{HaCaT}$ cells treated with IL-17A was quantified by real-time PCR. (C and D) A miR-146a-overexpressing vector (pmiR-146a) and a scrambled control hairpin in pCDHCMV-MCS-EF1-copGFP (pmiR) from SBI (System Biosciences) were used to generate stable miR-146a-overexpressing and control cell clones, respectively. The secretion of cytokines (IL-6 and IL-8) by HaCaT cells stably transfected with pmiR or pmiR-146a vectors was measured 2 days after stimulation with 25 or $100 \mathrm{ng} / \mathrm{mL}$ IL-17A. Three independent biological replicates were performed for the real-time PCR analysis. All results are presented as mean \pm SD. For statistical analysis, unpaired Student's $t$ test (B) or 2-way ANOVA with Tukey's multiple-comparison test (C and $\mathbf{D})$ was performed. ${ }^{* *} P<0.01$, ${ }^{* * *} P<0.001$.

$\mathrm{IL}-17 \mathrm{~A}(\mathrm{ng} / \mathrm{mL})$

flammatory cytokines after stimulation by IL-17A, we examined activation of the MAPK and NF- $\kappa$ B pathways in miR-146-overexpressing keratinocytes. As shown in Figure 4B, the MAPK/ERK pathway was highly activated by IL-17A in these cells, compared with control cells. We also found that ERK phosphorylation was significantly enhanced (more than 4 -fold, $P<0.01$; Figure $4 \mathrm{~B}$ ). In line with the data from galectin-7-knockdown cells, there were no obvious differences in the phosphorylation of NF- $\kappa \mathrm{B}$ and I $\mathrm{B} \alpha$ or their total protein levels between miR-146a-overexpressing cells and control cells (Figure 4B). Collectively, these results suggest that miR-146a promotes IL-17A-induced production of IL-6 and IL-8 through the MAPK/ERK pathway.

Galectin-7-deficient mice exhibit heightened IL-23-induced epidermal hyperplasia and skin inflammation. To further assess the participation of galectin-7 in the regulation of inflammatory responses in vivo, we studied galectin-7-deficient mice to determine the role of galectin-7 in IL-23-induced skin inflammation. In response to IL-23 stimulation, WT mice showed ear swelling resulting from hyperplasia of epidermal keratinocytes and leukocyte infiltration, as revealed by H\&E staining (Figure 5A). Skin sections from galectin-7-deficient mice showed a greater increase in ear and epidermal thickness compared with their WT control littermates 14 days after IL-23 injection (Figure 5, B and C). In addition, the numbers of infiltrating leukocytes were significantly higher in galectin-7-deficient mice (Figure 5D). IL-19 is known as a robust marker reflecting the magnitude of IL-23/IL-17 signaling in chronic inflammation (22). In microarray analysis, we also found that the amounts of proinflammatory cytokine mRNAs (IL-17A, CXCL5, and IL-19) were elevated in galectin-7-deficient mice, compared with WT mice (Supplemental Table 3). Taken together, these results indi- cate that galectin-7 has a suppressive effect on keratinocytemediated inflammatory responses.

Live deep-tissue visualization of galectin-7 expression in EGFPgalectin-7 transgenic mice by 2-photon microscopy. To examine the expression of galectin-7 in the skin during IL-23-induced inflammatory response in vivo, we generated an EGFP-galectin-7 fusion transgenic mouse model. This involved CRISPR/Cas9-mediated homologous recombination, which allowed insertion of an EGFP gene to fuse with the endogenous galectin-7 gene, where the expression of the fusion protein is under control of the native galectin-7 promoter. EGFP-galectin-7 mice exhibited an intense green fluorescence signal on the entire body surface (Figure 6A). In the $3 \mathrm{D}$ images obtained by 2-photon microscopy (Figure 6B), the green fluorescence signal was found to be confined to keratinocytes and hair follicles (Figure 6C) (the projected green signals from hair follicles are the reflected light from the hair). Immunoblot analysis indicated that EGFP-galectin-7 protein is expressed in the ear skin, dorsal skin, and esophagus, but not in the lung, kidney, liver, intestine, and colon (Figure 6D and our unpublished observations). In addition, we found no green fluorescence signals in bone marrow cells from these transgenic mice (Figure $6 \mathrm{E})$. We further performed affinity purification by using lactoseSepharose beads and demonstrated that EGFP-galectin-7 from the skin lysates retained lactose-binding activity (Figure 6F).

We performed daily intradermal IL-23 injections on the ears of these transgenic mice for 14 days, with the contralateral ear being injected with PBS as control. Compared with the control, the ear that received IL-23 had a profound thickening of the epidermis. We also injected Evans blue dye through the tail vein for visualization of the blood vessels (red fluorescence), and found that galectin-7 was not expressed in endothelial cells, since 
A



B

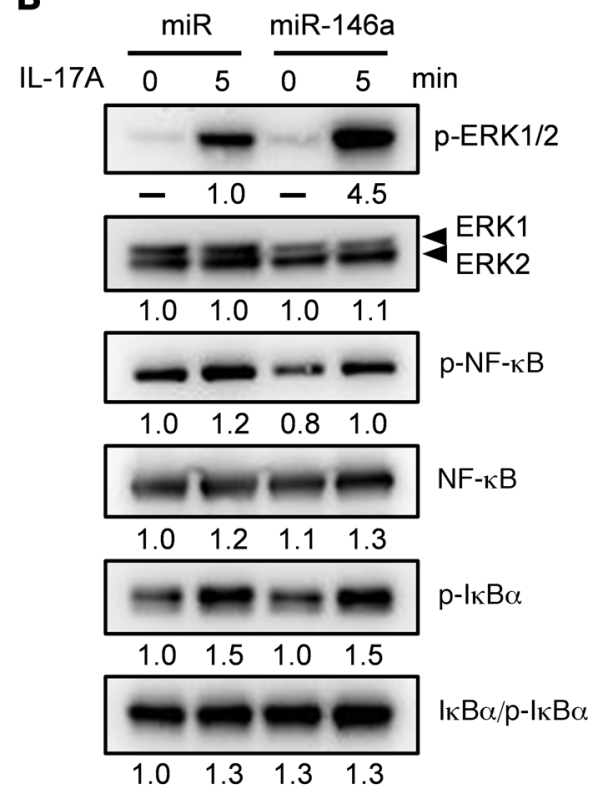

Figure 4. Reduced galectin-7 expression and miR-146a overexpression promote ERK1 and ERK2 signaling pathways triggered by IL-17A.

(A) Galectin-7-knockdown HaCaT cells and control cells were treated with IL-17A for 5 minutes, and cell lysates were analyzed by immunoblotting. Total ERK1, ERK2, NF- $\kappa B$, and $1 \kappa B \alpha$ and their phosphorylated forms were detected with the corresponding antibodies. (B) HaCaT cells stably transfected with pmiR (control vector) or pmiR-146a were treated with IL-17A for 5 minutes. Immunoblotting was performed as described in $\mathbf{A}$. Protein quantification data on phospho-ERK1 (p-ERK1) and phospho-ERK2 ( $p$-ERK2) were normalized to the control group in $\mathbf{A}$ and the miR group in $\mathbf{B}$. Data on total protein

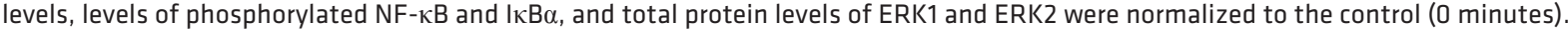

there were no green fluorescence signals in the blood vessel areas, as well as other areas in the dermis, in IL-23-treated skin (Supplemental Figure 7A). We further performed analysis of 3D images obtained on days $0,7,14$, and 21 , and showed an increase in the epidermal thickness in the IL-23-injected ear skin along with reduced green fluorescence signals on days 14 and 21 (Supplemental Figure 7B).

Fluvastatin increases galectin-7 levels and suppresses production ofproinflammatory cytokines in keratinocytes, and attenuates IL-23induced epidermal thickness in vivo. To use our observations on the antiinflammatory and antiproliferative effects of galectin-7, we scrutinized microarray databases for approved drugs that can induce galectin-7 expression. Four compounds were identified by connectivity map (CMap) data analysis: methylprednisolone, tobramycin, fluvastatin, and pempidine. In our microarray and proteomic data (our unpublished observations), we found that mRNA and protein levels of galectin-7 were negatively correlated with S100A7, which was reported to be upregulated in psoriatic keratinocytes (23). Among the predicted drugs, only fluvastatin induced galectin-7 mRNA and protein expression (Figure 7A and Supplemental Figure 8A) and reduced S100A7 mRNA expression (Figure 7B) in our in vitro experiments using $\mathrm{HaCaT}$ cells. In addition, we found that fluvastatin can induce $\mathrm{p} 21$ overexpression as revealed by immunoblot analysis (Supplemental Figure $8 \mathrm{~B})$. We next examined the influence of other statin drugs and found that atorvastatin, cerivastatin, pitavastatin, lovastatin, mevastatin, and simvastatin all induced galectin-7 expression in both HaCaT and HEKn cells (Supplemental Figure 8, C and D), while pravastatin was the least able to enhance galectin-7 expression (Supplemental Figure 8, C and D).
We found that fluvastatin attenuated IL-17A-induced IL- 6 and IL-8 secretion in HaCaT cells (Figure 7, C and D). We also observed that fluvastatin enhanced galectin-7 expression above basal levels, even in the presence of cytokines (TNF- $\alpha$ and IL-17A) that suppress galectin-7 expression (Figure 7E). In vivo, fluvastatin attenuated IL-23-induced ear skin thickening and epidermal hyperplasia (Figure 7F and Supplemental Figure 9, A and B). Consistent with our finding that pravastatin was barely able to enhance galectin-7 expression, it also had little effect on skin thickening in the same model (Figure 7F and Supplemental Figure 9C). We further tested whether the suppression of ear thickening by fluvastatin was dependent on galectin-7 by using galectin-7-deficient mice. We found that fluvastatin failed to suppress ear thickening in galectin-7-deficient mice (Supplemental Figure 10), suggesting that fluvastatin's amelioration of skin thickening was dependent on the expression of galectin-7. Thus, fluvastatin is a potent galectin-7 inducer that can suppress IL-17A- and IL-23-induced cytokine production and epidermal hyperplasia.

\section{Discussion}

We conclude that galectin-7 is downregulated in the keratinocytes of psoriatic human skin. This phenomenon was also confirmed in a mouse model of psoriasis. Mechanistically, we linked this to the action of IL-17A and TNF- $\alpha$ on keratinocytes, as demonstrated with the HaCaT and HEKn cell lines. By using both cell lines and a mouse psoriasis model, we were able to link the downregulated expression of galectin-7 to 2 cardinal histopathological features of psoriasis: keratinocyte hyperproliferation and skin inflammation. The former is consistent with our previous demonstration of the antiproliferative function of intracellular galectin-7 in keratino- 
A

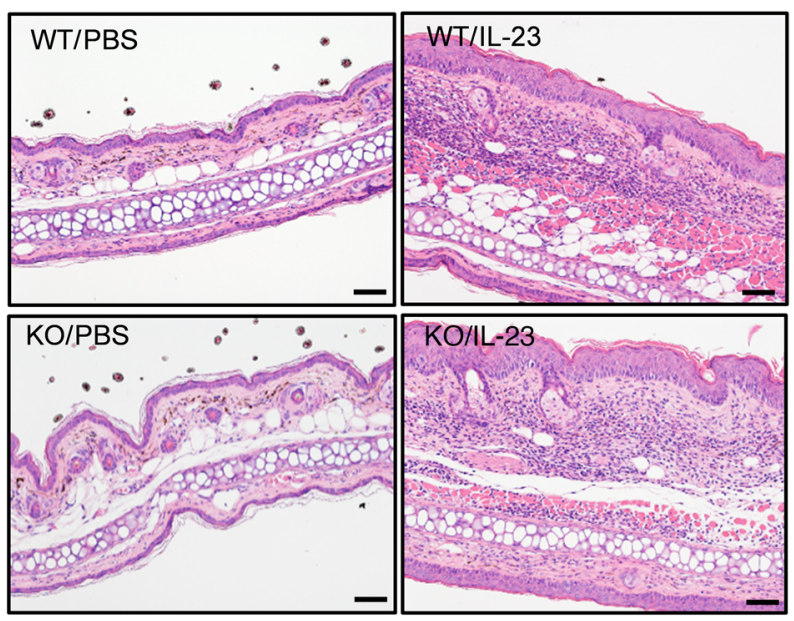

B

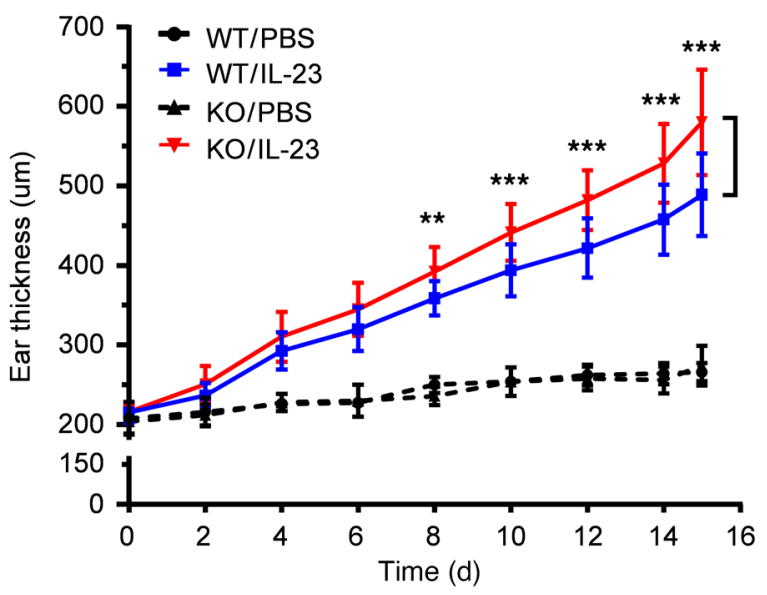

C

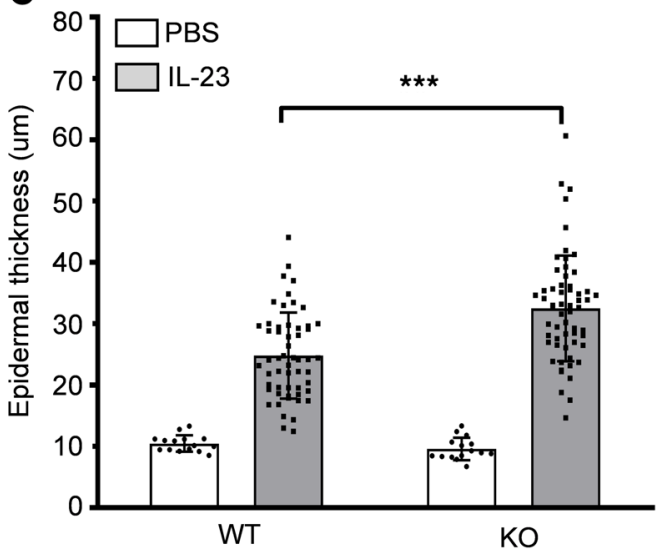

D

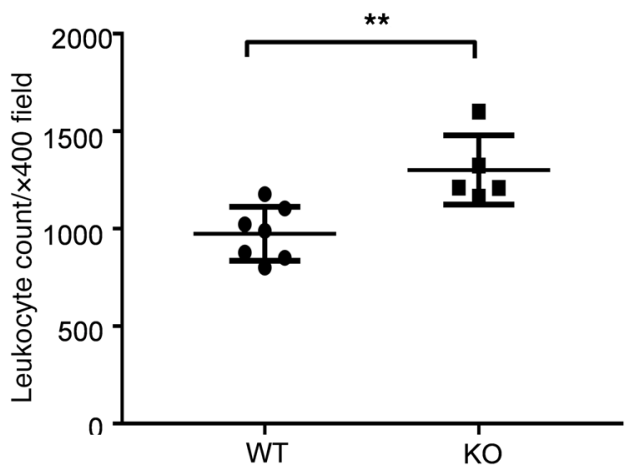

Figure 5. Galectin-7-deficient mice exhibit hyperproliferative keratinocytes and increased leukocyte infiltration at the intradermally IL-23-injected sites, as compared with their littermate controls. (A) H\&E staining of ear sections from WT or galectin-7-deficient (knockout; KO) mice injected intradermally with PBS or IL-23 every other day for 15 days. Scale bars: $50 \mu \mathrm{m}$. (B) Ear thickness of WT and galectin-7-deficient (KO) mice was measured before each intradermal injection, and measurements were taken at the center of the ears (WT/PBS, $n=5 ; \mathrm{WT} / \mathrm{IL}-23, n=18 ; \mathrm{KO} / \mathrm{PBS}, n=5 ; \mathrm{KO} / \mathrm{IL}-23, n=19$ ). For statistical analysis, ear thickness of KO/IL-23 was compared with that in the corresponding WT/IL-23 group at each time point. (C) Epidermal thicknesses of WT and KO mice from H\&E-stained sections as described in $\mathbf{A}$ obtained on day 15 from the same mice as described in $\mathbf{B}$. For each tissue section, 3 measurements were taken. (D) Leukocytes were counted in $\times 400$ magnified visual fields of tissue sections from IL-23-injected mice using the cell counting module in Imagej software (WT, $n=7 ; \mathrm{KO}, n=5$ ). All results are presented as mean \pm SD. For statistical analysis, 2-way ANOVA with Tukey's multiplecomparison test (B and $\mathbf{C}$ ) or unpaired Student's $t$ test $(\mathbf{D})$ was performed. ${ }^{* *} P<0.01,{ }^{* *} P<0.001$.

cytes (6). The latter is associated with the ability of intracellular galectin-7 to suppress IL-6 and IL-8 production in keratinocytes treated with IL-17A, as shown in this study. Moreover, we were able to link the antiinflammatory function of galectin- 7 to its ability to suppress the MAPK pathway. In the skin, galectin-7 is expressed exclusively in keratinocytes. We thus believe that downregulated expression of galectin-7 in psoriasis contributes to the pathogenesis solely through the dysregulated functions of lesional keratinocytes resulting from the deficiency of this protein.

Galectin-7, like other galectins, is synthesized without a classical leader peptide for protein secretion through the classical pathway. The protein is abundantly expressed in the cytosol and enriched in the nuclei in some cell types under certain conditions. Nevertheless, the protein can be released by cells into the extracellular space through unconventional protein secretory pathways. Our previous work (6) and present study demonstrated that intracellularly in kera- tinocytes, this protein functions by regulating intracellular signaling mediators, including JNK1, ERK, and miRNA, independently of the carbohydrate-binding activity of the protein. Thus, the finding in an earlier study (15) that there was no alteration in the levels of glycoligands in psoriasis lesions does not contradict our conclusions.

We previously found that galectin-7 regulates JNK1 stability, thereby controlling miR-203 expression. In the present study, we demonstrated that galectin-7 suppresses miR-146a expression, which, in turn, promotes ERK signaling during stimulation with IL-17A. We believe that there are 2 independent pathways regulating the expression of miR-203 and miR-146a, respectively. One piece of evidence we have is that we did not observe crosstalk between miR-203 knockdown and miR-146a overexpression in cells. Indeed, when we knocked down or overexpressed miR203, we did not observe any effect on cytokine production, nor feedback regulation on the miR-146a level. On the other hand, in 
A

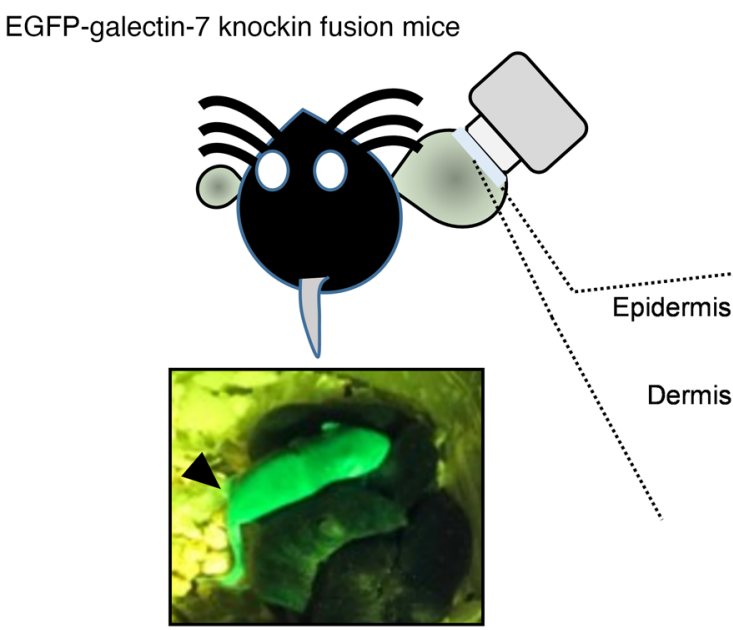

\section{B}

Two-photon fluorescence microscopy

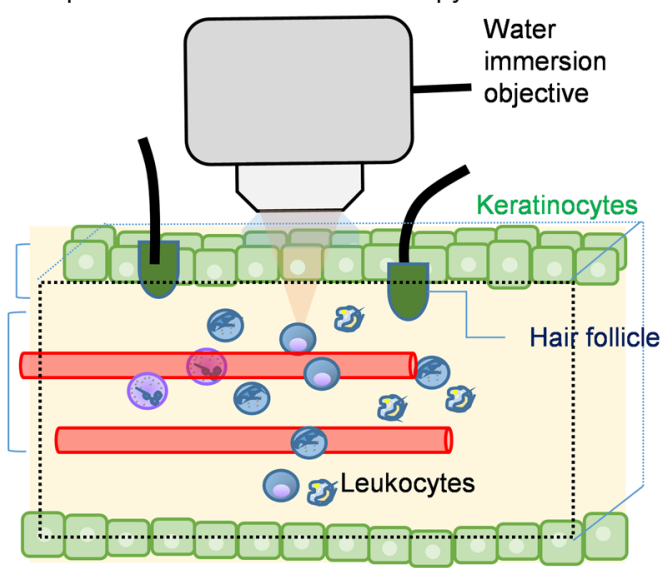

C
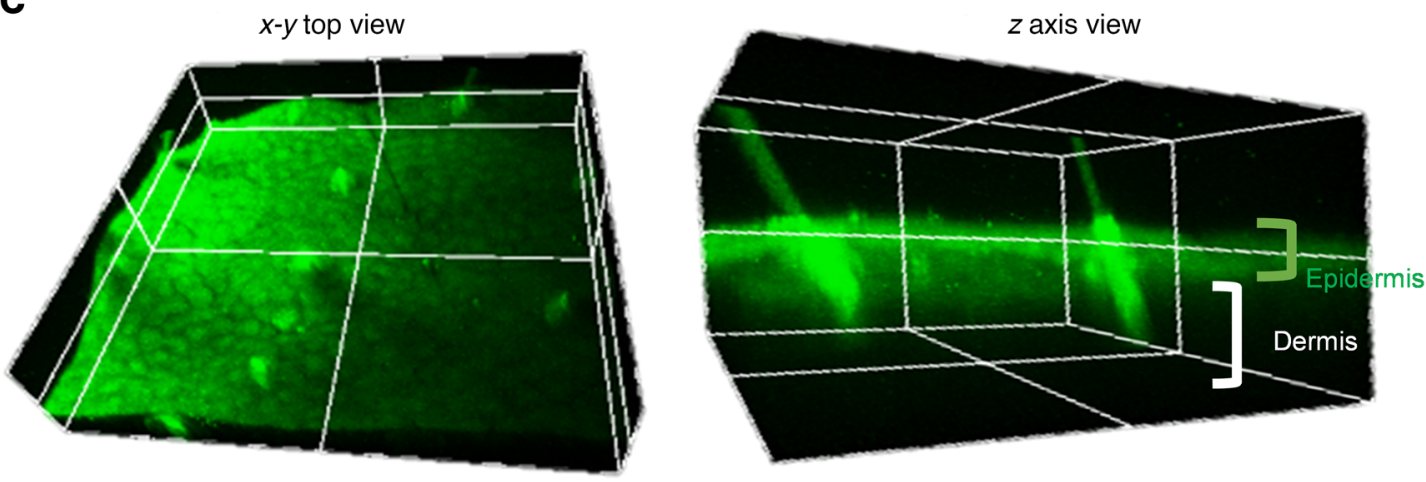

D

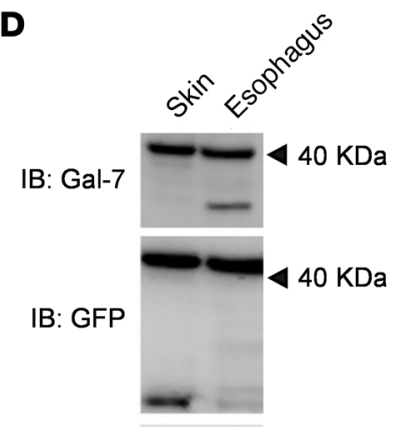

IB: Tubulin

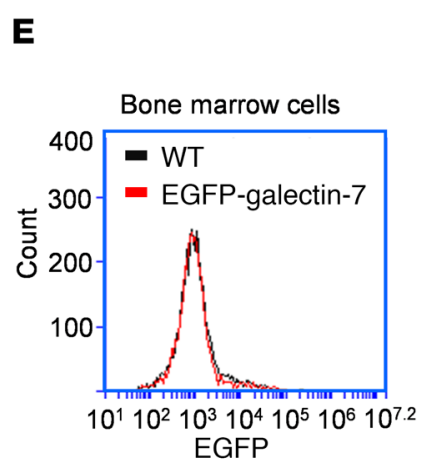

$55 \mathrm{KDa}$
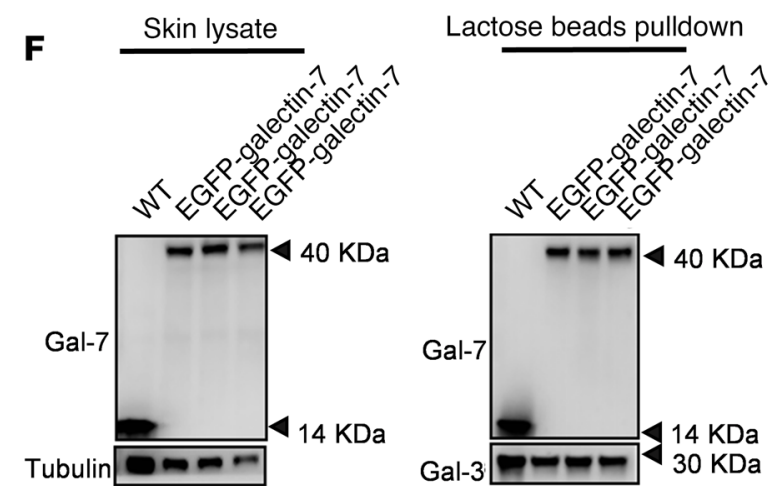

Figure 6. Two-photon deep-tissue visualization of EGFP-galectin-7 expression in transgenic mice. (A) Detection of green fluorescent signal on the whole-body surface. (B) Schematic representation of the 2-photon image analysis of deep tissue in the ear. (C) Three-dimensional images of the view of $x-y$ axis and $z$ axis planes. (D) Immunoblot analysis of galectin-7-GFP expression from tissues of EGFP-galectin-7 transgenic mouse. (E) Flow cytometry analysis of bone marrow cells purified from WT and EGFP-galectin-7 transgenic mice. (F) Affinity purification of EGFP-galectin-7 fusion protein from the lysates of skin samples from EGFP-galectin-7 transgenic mice, followed by immunoblot analysis.

miR-146a-overexpressing cells, we did not observe a change in their proliferation ability, but only cytokine production was elevated. Thus, we believe that although galectin-7 regulates these 2 miRNAs simultaneously, their effects are independent. The overall effect of galectin-7 deficiency through these 2 miRNAs is enhanced cell proliferation and cytokine production in keratinocytes, both of which lead to the psoriasis phenotype.
The single-nucleotide polymorphism rs2910164 in miR-146a was found to be associated with psoriasis through the regulation of EGFR expression (24). Overexpression of miR-146a in skin lesions was reported to correlate with clinical psoriasis scores (11). It is known that miR-146a is induced by zymosan through the NF-кB signaling pathway in keratinocytes. MiR-146a levels then soon decrease to a basal level because of negative feedback 
A

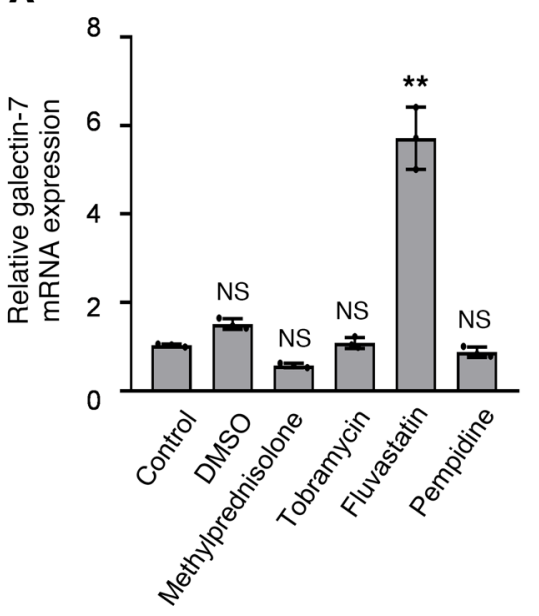

B

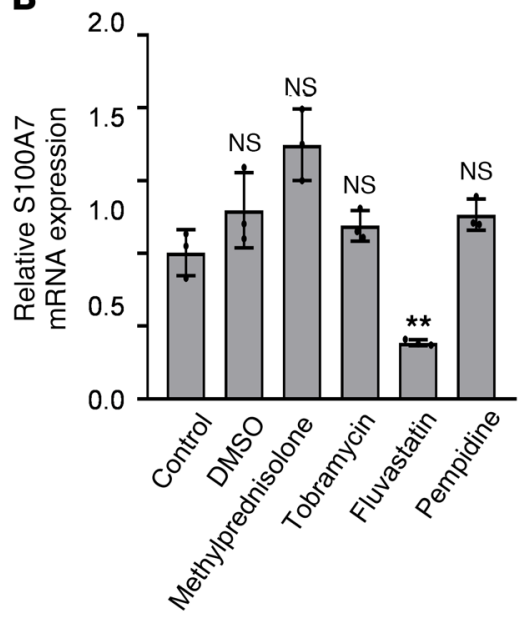

C

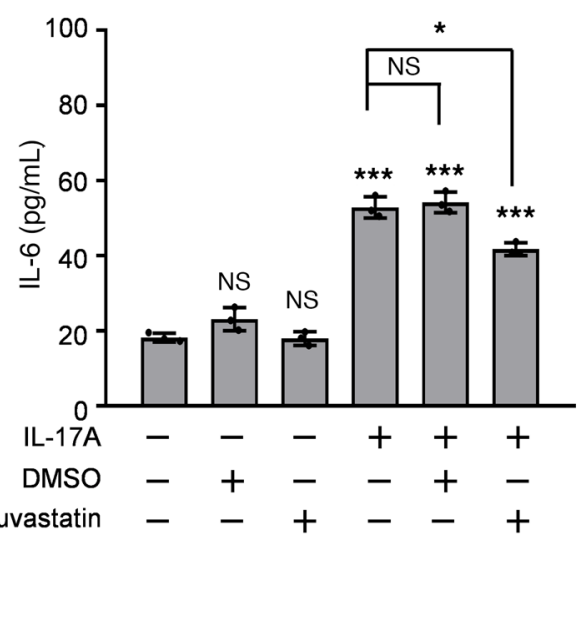

D

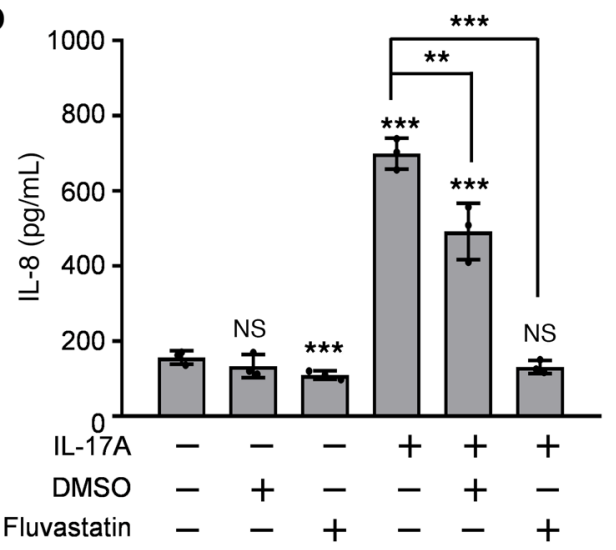

E



$\mathbf{F}$

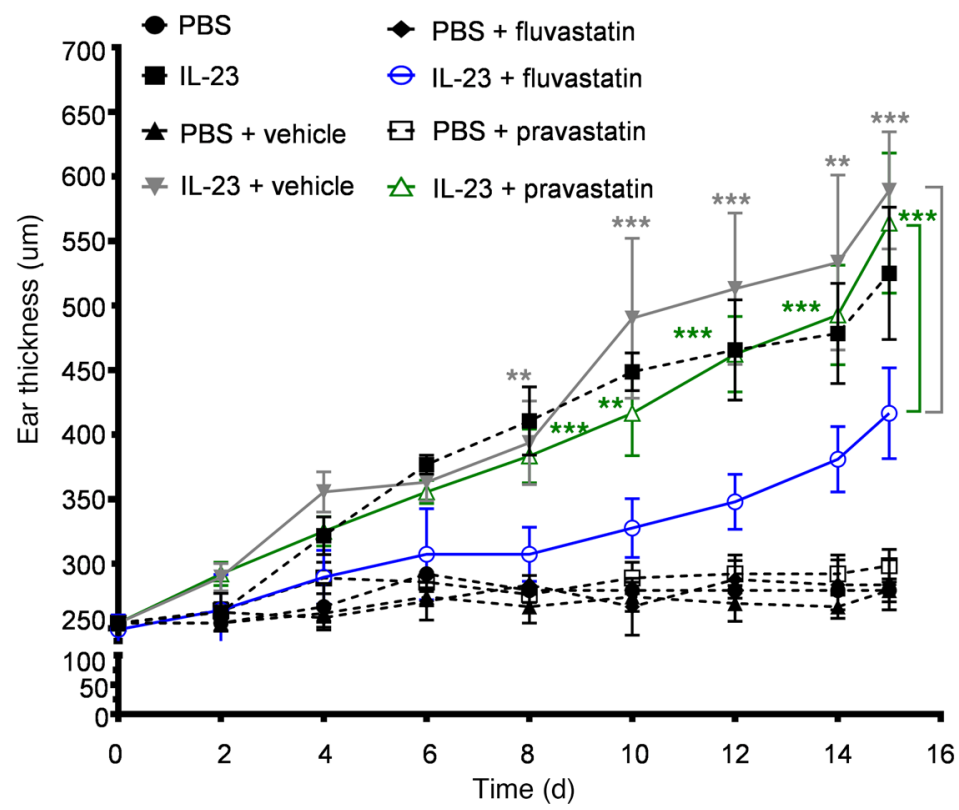

Figure 7. Fluvastatin increases galectin-7 levels in keratinocytes, suppresses production of proinflammatory cytokines, and attenuates IL-23induced epidermal thickness. (A and B) Real-time PCR analysis of the mRNA expression of galectin-7 and S100A7 in HaCaT cells treated with methylprednisolone $(10.6 \mu \mathrm{M})$, tobramycin $(8.6 \mu \mathrm{M})$, fluvastatin $(9.2 \mu \mathrm{M})$, pempidine $(13 \mu \mathrm{M})$, or vehicle control (DMSO) for 24 hours. The relative fold changes were calculated using the $\triangle \Delta \mathrm{Ct}$ method; data from all the samples were normalized to the control sample, and CAPDH served as an endogenous control. (C and D) Production of IL- 6 and IL-8 by HaCaT cells treated for 2 days with fluvastatin with or without IL-17A (200 ng/mL) was measured by ELISA. For statistical analysis, each group was compared with control group (first bar: group without IL-17A, DMSO, and fluvastatin). (E) Immunoblotting analysis of galectin-7 in keratinocytes treated with TNF- $\alpha$ or IL-17A with or without fluvastatin or DMSO. (F) Ear thicknesses of different groups - PBS $(n=3)$, IL-23 $(n=3)$, PBS + vehicle $(n=5)$, IL-23 + vehicle $(n=5)$, PBS + fluvastatin $(n=3)$, IL-23 + fluvastatin $(n=5)$, PBS + pravastatin $(n=4)$, IL-23 + pravastatin ( $n=5)$ - of mice subjected to intradermal injections of IL-23 or PBS, as described in Figure 5 , and treated with fluvastatin (blue line), pravastatin (green line), or saline. For statistical analysis, either 1-way (A and B) or 2-way (C, D, and F) ANOVA with Tukey's multiple-comparison test was used. Three independent biological replicates were performed for real-time PCR and ELISA analyses. The results (adjusted $P$ values) of group pairs are annotated on the graph (IL-23 + fluvastatin group vs. corresponding IL-23 + vehicle group indicated in gray; IL-23 + fluvastatin group vs. corresponding IL-23 + pravastatin group indicated in green). ${ }^{*} P<0.05,{ }^{* *} P<0.01,{ }^{* *} P<0.001$.

signaling (25). In this case, miR-146a was reported to exert an antiinflammatory action in keratinocytes through its known targets, including IRAK1 and TRAF6 (which are also required for miR-146a expression), and subsequent suppression of IL-8 and CCL2O expression (25). In addition, upregulation of miR-146a in the skin of patients with psoriasis was believed to contribute to lymphocyte infiltration (26). In contrast, here we found that IL-17A induced miR-146a expression, and $\mathrm{HaCaT}$ cells overexpressing miR-146a produced larger amounts of the proinflammatory cytokines IL-6 and IL-8. Therefore, our demonstration of the effect of IL-17A/miR-146a signaling on the inflammatory response is opposite to that of TLR2/miR-146a signaling reported 
previously (25). Another study identified miR-146a in the suppression of IL-17-mediated skin inflammation in mice induced by topical application of imiquimod (27). In this model, the effect of miR-146a on skin inflammation may influence both immune cells and keratinocytes. Thus, our results and those of other researchers suggest that miRNAs may yield different responses in cells, depending on the experimental conditions, including culture conditions (e.g., growth factors), cell type, and activation status. Mechanistically, miRNA functions are dependent on their target mRNA levels. Thus, the overexpression of miR-146a in psoriatic skin lesions might be related to its inability to inhibit target genes (11). In this case, transcriptomic analysis should provide further information, and this topic has yet to be explored.

Other studies revealed the participation of miRNA-203 in the modulation of the inflammatory response. For example, miR-203 can suppress the levels of IL-8 mRNA in keratinocytes treated with TNF- $\alpha(28,29)$, including levels of the suppressors of cytokine signaling (SOCS3 and SOCS6) in gingival epithelial cells (30). We tested whether galectin-7 affects the levels of SOCS1, SOCS3, and SOCS6 in keratinocytes by immunoblot analysis, but did not observe a consistent downregulation of these proteins in galectin-7-knockdown cells (our unpublished observations). However, we cannot entirely exclude the possibility that galectin-7 also regulates the inflammatory response through this miRNA.

Psoriasis is considered as a metabolic syndrome associated with an increased risk of cardiovascular diseases, including acute coronary syndrome, arterial hypertension, and myocardial infarction $(31,32)$. Patients with this condition are often found to also have higher blood levels of cholesterol and triglycerides. Accordingly, patients with psoriasis may benefit from statin drugs, a group of 3-hydroxy-3-methylglutaryl coenzyme A (CoA) reductase inhibitors commonly taken by patients with hypercholesterolemia resulting from abnormal lipid metabolism. Interestingly, the beneficial effects of statins on skin lesions in patients with psoriasis have already been documented and are possibly mediated by suppressed inflammation and improved skin regeneration (33-35). Our findings suggest that the beneficial effects of statins may be related to their upregulation of galectin-7 expression. Different statins may have different effects on psoriasis, and those that are stronger inducers of galectin-7 (such as fluvastatin and simvastatin) may be preferable over weaker inducers (such as pravastatin). Indeed, it has been reported that the former are more effective than the latter at inhibiting CCL20 production by human keratinocytes induced by IL-1 $\beta$, TNF- $\alpha$, or IL-17A (34). It is to be noted that a reduction in CCL20 expression can decrease the number of CCR6 ${ }^{+}$ Th17 cells, a cell type crucial for the development of psoriatic skin lesions. While we have yet to test whether galectin-7 can also suppress CCL20 production, our findings suggest that galectin-7 may be responsible for the specificity of statins in terms of regulating the production of proinflammatory cytokines in human skin, and thus may contribute to their therapeutic effects on psoriasis.

In summary, galectin-7 expression in keratinocytes is downregulated by cytokines responsible for the development of psoriasis. This resulted in the hyperproliferation of keratinocytes and skin inflammation. Approaches to induce galectin-7 in keratinocytes may be useful for the treatment of psoriasis. We have identified one such approach: the use of selected kinds of statins, which is intriguing, as psoriasis is considered a metabolic syndrome associated with dyslipidemia, and thus patients would benefit from the use of statins. Additional investigations along this approach have the potential to identify statins that can be selected on the basis of their potency in the induction of galectin-7 and will be more effective in the treatment of psoriasis.

\section{Methods}

Human skin tissue samples. Human skin tissues from patients with psoriasis $(n=27)$ and healthy volunteers $(n=75)$ were acquired with informed consent in accordance with a protocol approved by the Chung Shan Medical University Institutional Review Board. Lesional and nonlesional skin section pairs were acquired in accordance with protocols approved by the Institutional Review Boards of Chung Shan Medical School ( $n=5$ cases) and Taipei Tzu Chi Hospital, Buddhist Tzu Chi Medical Foundation ( $n=5$ cases), respectively. Patients with psoriasis vulgaris received a diagnosis based on clinical and histopathological criteria, and patients receiving systemic therapies were excluded. All patients were not treated with topical medications for at least 1 month before the biopsies were taken.

Mice. Galectin- $7^{+/-}$mice with a C57BL/6 background were generated by the European Conditional Mouse Mutagenesis Program (EUCOMM) from the embryonic stem cell line EPD0327_3_B05 and were acquired from the Wellcome Trust Sanger Institute (Cambridge, United Kingdom). We implemented a breeding scheme of heterozygotes crossed with heterozygotes, to generate WT, heterozygous, and homozygous mice. All the mice in this study were genotyped, and control WT mice were littermates of the homozygous mice. Both male and female mice 6-8 weeks old were used in the experiments.

Keratinocyte culture and preparation of galectin-7-knockdown cells. The human keratinocyte cell line $\mathrm{HaCaT}$ was acquired as described in our previous publication (6), and the cells were maintained at $37^{\circ} \mathrm{C}$ in DMEM (Gibco) supplemented with 10\% FBS, 2 mM glutamine, $100 \mathrm{U} / \mathrm{mL}$ penicillin (MilliporeSigma), and $100 \mu \mathrm{g} / \mathrm{mL}$ streptomycin (Gibco). Short hairpin RNA (shRNA) reagents were obtained from the National RNAi Core Facility located at the Institute of Molecular Biology and Genomic Research Center, Academia Sinica, supported by the National Core Facility Program for Biotechnology Grants of NSC (NSC 100-2319-B-001-002). Four shRNAs with clone IDs of TRCN0000057393, TRCN0000057394, TRCN0000057395, and TRCN0000057396 were used to generate stable galectin-7knockdown HaCaT clones. HaCaT cells were infected with 1 of the 4 shRNA-expressing lentiviruses, and puromycin was used to select stable cell clones after 3 days of infection. After puromycin selection, 4 individual pooled clones were cultured in puromycin-free medium for 2 weeks before further analysis.

Primary neonatal human epithelial keratinocytes (HEKn cells) were purchased from Gibco (catalog C-001-5C). HEKn cells were cultured at $37^{\circ} \mathrm{C}$ in keratinocyte serum-free medium (K-SFM) supplemented with $30 \mu \mathrm{g} / \mathrm{mL}$ bovine pituitary extract and $5 \mathrm{ng} / \mathrm{mL}$ recombinant human epidermal growth factor obtained from the same manufacturer. Compared with HaCaT cells, HEKn cells have a limited proliferation ability and are not suitable for establishing shRNA-based stable knockdown cells; therefore, we used a small interfering RNA (siRNA) oligonucleotide to transiently knock down the expression of galectin-7. The Silencer Select siRNAs were purchased from Invitrogen, including negative control 1 and a predesigned siRNA targeting 
human galectin-7 (catalog s230574-75-76). HEKn cells were treated with 30 pmol of siRNAs in a 6-well plate with $1 \times 10^{6}$ cells per well using Lipofectamine 2000 (Invitrogen) as a transfection agent.

Measurement of cytokine production. To measure the concentrations of proinflammatory cytokines secreted by keratinocytes, we seeded $2 \times 10^{4}$ cells per well in 96 -well plates. Cells were incubated for 24 hours and then treated with $50 \mu \mathrm{g} / \mathrm{mL}$ LPS (MilliporeSigma, catalog L4391), $100 \mathrm{ng} / \mathrm{mL}$ human TNF- $\alpha$ (PeproTech, catalog 30001), 100 or $200 \mathrm{ng} / \mathrm{mL}$ human IL-17A (PeproTech, catalog 200-17), 200 ng/mL human IL-22 (PeproTech, catalog 200-22), $100 \mathrm{ng} /$ $\mathrm{mL}$ human IL-23 (PeproTech, catalog 200-23), or $50 \mathrm{ng} / \mathrm{mL} \mathrm{IFN- \gamma}$ (eBioscience, catalog 39-8319-65) for 48 hours at $37^{\circ} \mathrm{C}$. In addition, the MEK inhibitor PD98059 (Merck Millipore Calbiochem) was added along with stimulation by LPS or IL-17A (48 hours). The supernatants were then collected and analyzed by ELISAs, which were performed using specific combinations of paired antibodies (capture and detection antibodies).

Immunoblot analysis. Cells were harvested and lysed in RIPA lysis buffer containing $1 \%$ Triton X-100 and a protease inhibitor cocktail (MilliporeSigma), and the total protein concentrations were measured with a Bradford Protein Assay Kit (Bio-Rad). Proteins in the samples of the lysates with the same amounts of total protein were separated by SDS-PAGE and analyzed by immunoblotting. Primary antibodies against phosphorylated ERK1 (pT202)/ERK2 (pT185) (E337, Epitomics, catalog 1481-1), ERK1 (EP4967, Epitomics, catalog 3739-1), ERK2 (E460, Epitomics, catalog 1586-1), NF-кB p65 (E379, Abcam, catalog ab32536), phospho-NF-кB p65 (pS536) (EP2294Y, Abcam, catalog

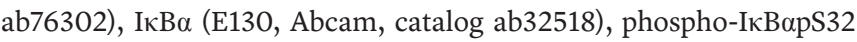
(EPR3148, Abcam, catalog ab92700), $\beta$-actin (AC-15, MilliporeSigma, catalog 5441), or GAPDH (EPR1977Y, Epitomics, catalog 2251-1) were applied to detect the corresponding proteins (Supplemental Table 4). Goat anti-galectin-7 antibodies were used as described previously (3). HRP-conjugated secondary antibodies against mouse, rabbit, or goat IgG were then incubated with the membranes, and the proteins were visualized by chemiluminescence according to the manufacturer's instructions (MilliporeSigma, Advanta). Protein quantification was performed using Soft MaX Pro 6.2.1 software.

Quantitative real-time PCR. Total RNAs (including mRNA and miRNA) were extracted from cultured cells or mouse ears using TRIzol Reagent (Invitrogen). For measurement of human galectin-7, IL-6, IL-8, IL-17A, and IFN- $\gamma$ mRNA levels, mRNAs were reversetranscribed into cDNA by means of the iScript cDNA Synthesis Kit (Bio-Rad), and real-time PCR was carried out, using specific Universal Probe Library probes (Roche) accompanied by primers targeting these gene products, according to the manufacturer's instructions.

For miR-146a quantification, total RNA samples were converted to $\mathrm{CDNA}$, and real-time PCR was conducted with specific primers targeting miR-146a using the Mir-X miRNA First-Strand Synthesis and SYBR qRT-PCR Kit (Clontech). Human GAPDH and U6 snRNA were selected as internal controls for normalization of the levels of mRNAs and miRNAs, respectively. Relative levels of mRNAs and miRNAs were calculated, and fold changes were obtained using the $\Delta \Delta \mathrm{Ct}$ method and compared with vector-only control cells.

IL-23-induced mouse model of psoriasis. The IL-23-induced mouse psoriasis-like model was established as previously described (17). Intradermal injection of recombinant mouse IL-23 (1 $\mu \mathrm{g}$; eBioscience, catalog 34-8231-85) (or PBS as control) into the right ear of anesthe- tized mice was performed using an insulin syringe every other day for 14 days. Ear thickness was measured before the intradermal injection, and measurements were taken at the center of the ears using a pocket thickness gauge (Mitutoyo Corp.). Mice were euthanized on day 15; the ears were collected, and half of the ears were embedded in paraffin for $\mathrm{H} \& \mathrm{E}$, immunohistochemical, and in situ hybridization staining, while RNAs from the other half of the ears were extracted for analysis of the cytokine and miRNA profiles by quantitative real-time PCR.

To study the effect of statins in this mouse psoriasis model, 30 $\mathrm{mg} / \mathrm{kg} / \mathrm{d}$ fluvastatin (Cayman, 10010337) and pravastatin (Cayman, 10010343) were administrated by oral gavage to the mice once daily for 14 days. Saline was used as a control. The experimental groups were designed and arranged as follows: PBS $(n=2), \mathrm{IL}-23(n=3), \mathrm{PBS}$ + saline $(n=5), \mathrm{IL}-23+$ saline $(n=5), \mathrm{PBS}+$ fluvastatin $(n=3), \mathrm{IL}-23$ + fluvastatin $(n=4)$, PBS + pravastatin $(n=4)$, and IL-23 + pravastatin $(n=4)$. Ear thickness was measured as described above. On day 15 , the mice were euthanized, and their ears were collected and processed as described above.

Histology and immunohistochemistry. Sections of paraffinembedded mouse ears were prepared and stained with H\&E by the Pathology Core Laboratory (Institute of Biomedical Sciences, Academia Sinica). For immunohistochemical staining, 5- $\mu$ m-thick sections of paraformaldehyde-fixed mouse ears or human skin were deparaffinized and hydrated with distilled water. Heat-induced epitope retrieval was performed by incubation in citrate buffer (Thermo Fisher Scientific) at $98^{\circ} \mathrm{C}$ for 10 minutes, and endogenous peroxidase was quenched by treatment with $3 \% \mathrm{H}_{2} \mathrm{O}_{2}$ in PBS for 5 minutes. Next, $2.5 \%$ horse serum (Vector Laboratories) was applied for 1 hour to block nonspecific binding on the tissue sections. Galectin-7 in both mouse and human skin was detected by incubation with a goat anti-galectin-7 antibody (3) (primary antibody) for 1 hour. After washing with PBS, the histological slides were incubated with a polymer-HRP-conjugated horse anti-goat IgG antibody (Vector Laboratories) (secondary antibody) for 30 minutes. The staining reaction for galectin-7 was visualized by means of the ImmPACT DAB Peroxidase Substrate Kit (Vector Laboratories), and positive signals of DAB chromogen were developed as red-brown precipitates. The slides were counterstained with hematoxylin (Vector Laboratories) to detect nuclei. Quantification of immunohistochemical staining was performed by computer-assisted methods. The $\times 100$ magnified visual fields of the tissue sections (total area of $1.5 \mathrm{~mm}^{2}$ ) were examined. The epidermal region of each visual field was selected, and the intensity of galectin-7 staining from each pixel in the epidermis region was quantified using ImageJ software (NIH). To calculate the mean intensity of galectin-7 staining in each skin section, the intensity of galectin-7 staining was divided by the size of the epidermal area observed.

In situ hybridization of miRNA. LNA-modified, digoxigeninlabeled DNA probes complementary to human miR-146a (Exiqon) were designed for in situ hybridization of mouse and human skin sections according to the manufacturer's instructions. A scrambled miRNA probe was a negative control, and the probe complementary to U6 snRNA served as a positive control. All the sections were deparaffinized in xylene and rehydrated in a graded series of ethanol solutions. For reagent penetration, the sections were subjected to proteinase $\mathrm{K}$ digestion $(5-10 \mu \mathrm{g} / \mathrm{mL})$ for 5 minutes at $37^{\circ} \mathrm{C}$, followed by treatment with $4 \%$ paraformaldehyde in PBS for 15 minutes, and 
with a prehybridization solution (Biochain) at $50^{\circ} \mathrm{C}$ for $3-4$ hours. The tissue samples were hybridized with specific probes overnight at $53^{\circ} \mathrm{C}$. After the sections were washed with an SSC buffer (Biochain) dilution series, immunological detection with an alkaline phosphatase-conjugated anti-digoxigenin antibody (Biochain) was carried out. Detection of signals was based on a reaction with nitro blue tetrazolium chloride/5-bromo-4-chloro-3-indolyl phosphate substrate (Biochain), followed by counterstaining of nuclei with nuclear fast red (Merck Millipore).

Generation of EGFP-galectin-7 transgenic mice. To generate mice expressing EGFP fused to the N-terminus of galectin-7, we designed an sgRNA targeted to the start codon of the galectin-7 gene, and used an EGFP-galectin-7 sequence plasmid (pcDNA3.1 with a 500-bp upstream and downstream galectin-7 sequence near the start codon and a linker GGGSG added to the C-terminus of the EGFP gene) as a homologous recombination repair template. The galectin-7 gene locus of CL57BL6 is located on chromosome $7: 28,863,853-28,866,284$. The sgRNA target sequence of ENSMUSG00000053522 is GCCATGTCTGTAAGTATACT. The founder mice $\left(\mathrm{F}_{0}\right)$, expressing a high green fluorescence signal on the whole-body surface, were selected and genotyped by PCR. The $F_{1}$ of the founders were then crossed to acquire homo-transgenic mice $\left(\mathrm{F}_{2}\right)$. Both male and female mice 6-8 weeks old were used in the experiments.

Two-photon microscopy. To visualize fluorescence signals from the deep tissue of the ear in live mice, we used an FVMPE-RS multiphoton laser scanning microscope (Olympus), equipped with Chameleon Vision II laser (Coherent). We used the excitation wavelength of $850 \mathrm{~nm}$ and green filter (495-540 nm) for GFP, and a red filter (575$645 \mathrm{~nm}$ ) for red fluorescence signal. The $\times 25$ objective (XLPLN25xWMP2) was used for this experiment. For live animal imaging experiments, we anesthetized mice with isoflurane anesthesia and located the mice on a stage heated to $37^{\circ} \mathrm{C}$. The image data were further analyzed using Image J software.

Statistics. All quantitative data are presented as mean \pm SEM, SE, or SD unless indicated otherwise. We performed 2-tailed unpaired or paired Student's $t$ test. Either 1-way ANOVA or 2-way ANOVA with Tukey's multiple-comparison test was performed as described in the figure legends to compare the groups of samples, and differences with $P$ values less than 0.05 were considered statistically significant. For in vivo studies, the statistical analyses were performed according to the description in the figure legends.

Study approval. The analysis of human tissue samples was approved by the Institutional Review Board of Academia Sinica, Taipei, Taiwan [AS-IRB01-16076 (N)]. All animal experimental procedures were approved by the Animal Care and Use Committee of the Institute of Biomedical Sciences (IACUC Protocol ID: 10-11-090, Academia Sinica, Taipei, Taiwan).

\section{Author contributions}

FTL, CHL, and HLC designed the research studies. HLC, CCH, CHL, MPL, PYH, CSC, and DYC conducted experiments and acquired and analyzed data. DKH, YHL, and PC provided valuable suggestions on the projects and manuscript. YHL, YPH, and TNL provided crucial reagents and materials. FTL, HLC, DKH, and CHL wrote the manuscript.

\section{Acknowledgments}

We thank N. Fusenig (Deutsche Krebsforschungszentrum, Heidelberg, Germany) for providing the HaCaT cell line. We thank Emanual Maverakis (Department of Dermatology, School of Medicine, University of California, Davis, Sacramento, California) for discussion of psoriasis pathology. This research was supported by the Summit Program, Academia Sinica (AS-SUMMIT-109), Ministry of Science and Technology (AS-KPQ-109-BioMed and MOST 109-0210-01-18-02); and by the Ministry of Science and Technology (MOST 104-0210-01-09-02, MOST 105-0210-01-13-01, and MOST 106-0210-01-15-02). We acknowledge the Transgenic Core Facility of Academia Sinica for consultation and generation of EGFP-galectin-7 transgenic mice. The Core was funded by the Academia Sinica Core Facility and Innovative Instrument Project (AS-CFII-108-107). We thank the National Laboratory Animal Center, NARLabs, Taiwan, for technical support in mouse breeding.

Address correspondence to: Fu-Tong Liu, 128 Academia Road, Section 2, Nankang, Taipei 11529, Taiwan. Phone: 886.2.2652.3001; Email: ftliu@ibms.sinica.edu.tw. Or: Department of Dermatology, University of California, Davis, School of Medicine, $3301 \mathrm{C}$ Street, Suite 1400, Sacramento, California 95816, USA. Phone: 916.734.6377; Email: fliu@ucdavis.edu.
1. Polyak K, Xia Y, Zweier JL, Kinzler KW, Vogelstein B. A model for p53-induced apoptosis. Nature. 1997;389(6648):300-305.

2. Bernerd F, Sarasin A, Magnaldo T. Galectin-7 overexpression is associated with the apoptotic process in UVB-induced sunburn keratinocytes. Proc Natl Acad Sci U S A. 1999;96(20):11329-11334.

3. Kuwabara I, et al. Galectin-7 (PIG1) exhibits pro-apoptotic function through JNK activation and mitochondrial cytochrome c release. J Biol Chem. 2002;277(5):3487-3497.

4. Magnaldo T, Bernerd F, Darmon M. Galectin-7, a human 14-kDa S-lectin, specifically expressed in keratinocytes and sensitive to retinoic acid. Dev Biol. 1995;168(2):259-271.

5. Gendronneau G, et al. Galectin-7 in the control of epidermal homeostasis after injury. Mol Biol Cell. 2008;19(12):5541-5549.
6. Chen HL, et al. Galectin-7 regulates keratinocyte proliferation and differentiation through JNK-miR-203-p63 signaling. J Invest Dermatol. 2016;136(1):182-191.

7. Xia J, Zhang W. MicroRNAs in normal and psoriatic skin. Physiol Genomics. 2014;46(4):113-122.

8. Zhao M, et al. Up-regulation of microRNA-210 induces immune dysfunction via targeting FOXP3 in CD4(+) T cells of psoriasis vulgaris. Clin Immunol. 2014;150(1):22-30.

9. Meisgen F, et al. MiR-21 is up-regulated in psoriasis and suppresses T cell apoptosis. Exp Dermatol. 2012;21(4):312-314.

10. Xu N, et al. MiR-125b, a microRNA downregulated in psoriasis, modulates keratinocyte proliferation by targeting FGFR2. J Invest Dermatol. 2011;131(7):1521-1529.

11. Xia P, et al. Dysregulation of miRNA146a versus IRAK1 induces IL-17 persistence in the psoriatic skin lesions. Immunol Lett. 2012;148(2):151-162

12. Choy DF, et al. Comparative transcriptomic analyses of atopic dermatitis and psoriasis reveal shared neutrophilic inflammation. J Allergy Clin Immunol. 2012;130(6):1335-1343.e5.

13. Andrianne M, et al. Tristetraprolin expression by keratinocytes controls local and systemic inflammation. JCI Insight. 2017;2(11):e92979.

14. Swindell WR, et al. RNA-seq identifies a diminished differentiation gene signature in primary monolayer keratinocytes grown from lesional and uninvolved psoriatic skin. Sci Rep. 2017;7(1):18045.

15. Lacina L, Plzáková Z, Smetana K Jr., Stork J, Kaltner H, André S. Glycophenotype of psoriatic skin. Folia Biol (Praha). 2006;52(1-2):10-15. 
16. Rizzo HL, Kagami S, Phillips KG, Kurtz SE, Jacques SL, Blauvelt A. IL-23-mediated psoriasis-like epidermal hyperplasia is dependent on IL-17A. JImmunol. 2011;186(3):1495-1502.

17. Chan JR, et al. IL-23 stimulates epidermal hyperplasia via TNF and IL-20R2-dependent mechanisms with implications for psoriasis pathogenesis. JExp Med. 2006;203(12):2577-2587.

18. Res PC, et al. Overrepresentation of IL-17A and IL-22 producing CD8 $\mathrm{T}$ cells in lesional skin suggests their involvement in the pathogenesis of psoriasis. PLoS One. 2010;5(11):e14108.

19. Arican O, Aral M, Sasmaz S, Ciragil P. Serum 1 evels of TNF-alpha, IFN-gamma, IL-6, IL-8, IL-12, IL-17, and IL-18 in patients with active psoriasis and correlation with disease severity. Mediators Inflamm. 2005;2005(5):273-279.

20. Martin DA, et al. The emerging role of IL-17 in the pathogenesis of psoriasis: preclinical and clinical findings. J Invest Dermatol. 2013;133(1):17-26.

21. Guttman-Yassky E, et al. Low expression of the IL-23/Th17 pathway in atopic dermatitis compared to psoriasis. J Immunol. 2008;181(10):7420-7427.

22. Witte E, et al. IL-19 is a component of the pathogenetic IL-23/IL-17 cascade in psoriasis. J Invest
Dermatol. 2014;134(11):2757-2767.

23. Sato Y, Kajihara I, Yamada-Kanazawa S, Jinnin M, Ihn H. S100A7 expression levels in coordination with interleukin-8 indicate the clinical response to infliximab for psoriasis patients. J Dermatol. 2017;44(7):838-839.

24. Zhang W, et al. A single-nucleotide polymorphism of miR-146a and psoriasis: an association and functional study. J Cell Mol Med. 2014;18(11):2225-2234.

25. Meisgen F, et al. MiR-146a negatively regulates TLR2-induced inflammatory responses in keratinocytes. JInvest Dermatol. 2014;134(7):1931-1940.

26. Sonkoly E, et al. MicroRNAs: novel regulators involved in the pathogenesis of psoriasis? PLOS One. 2007;2(7):e610.

27. Srivastava A, et al. MicroRNA-146a suppresses IL-17-mediated skin inflammation is genetically associated with psoriasis. J Allergy Clin Immunol. 2017;139(2):550-561.

28. Wei T, Xu N, Meisgen F, Stahle M, Sonkoly E, Pivarcsi A. Interleukin- 8 is regulated by miR-203 at the posttranscriptional level in primary human keratinocytes [published online April 19, 2013]. Eur J Dermatol. https://www.jle.com/10.1684/ ejd.2013.1997.
29. Yi R, Poy MN, Stoffel M, Fuchs E. A skin microRNA promotes differentiation by repressing 'stemness'. Nature. 2008;452(7184):225-229.

30. Moffatt CE, Lamont RJ. Porphyromonas gingivalis induction of microRNA-203 expression controls suppressor of cytokine signaling 3 in gingival epithelial cells. Infect Immun. 2011;79(7):2632-2637.

31. Paiva-Lopes MJ, Delgado Alves J. Psoriasisassociated vascular disease: the role of HDL. JBiomed Sci. 2017;24(1):73.

32. Choi YM, Famenini S, Wu JJ. Incidence of pulmonary arterial hypertension in patients with psoriasis: a retrospective cohort study. Perm J. 2017;21:16-073.

33. Greenwood J, Steinman L, Zamvil SS. Statin therapy and autoimmune disease: from protein prenylation to immunomodulation. Nat Rev Immunol. 2006;6(5):358-370.

34. Kim TG, Byamba D, Wu WH, Lee MG. Statins inhibit chemotactic interaction between CCL2O and CCR6 in vitro: possible relevance to psoriasis treatment. Exp Dermatol. 2011;20(10):855-857.

35. Namazi MR. Statins: novel additions to the dermatologic arsenal? Exp Dermatol. 2004;13(6):337-339. 